\title{
Research on Important Techniques of Independent Learning-Based Course and Self-Learning Terminal Platform in Electrical Engineering and Automation
}

\author{
PU Haitao \\ Department of Electrical Engineering \&Information \\ Technology \\ Shandong University of Science and Technology \\ Jinan, China \\ kdpht@163.com
}

\author{
DU Weixiu \\ College of Electrical Engineering \& Automation \\ Shandong University of Science and Technology \\ Qingdao, China \\ dweixiu@163.com
}

\begin{abstract}
Following with the gradually improvement of smart phone and the coming of Internet plus era, the learners can get rid of the temporal and spatial confinement, and learn with their own requirement anywhere, anytime through mobile terminal. In this paper, the specialized courses of Electrical Engineering and Automation in Shandong University of Science and Technology are taken as example, from the point of mobile learning and the characteristics of smart phone, Utilizing the Big Data analysis technique, through collecting the time, period, frequency, content, and extension of learning, which can be exploited to form the user behavioral database, construct the data model to implement the analysis and prediction, e.g. recommending the favorite courses to specific user, optimizing the courseware with amount of click rates, analyzing the completion level of learning task, and supplying with better selflearning resource to the students.
\end{abstract}

Keywords-Mobile Learning; Learning Terminal; Big Data; Electrical Engineering and Automation; Independent Learning component;

\section{INTRODUCTION}

In recent years, following the rapid development of mobilebased techniques, a novel remote distance learning pattern was produced, which is named after mobile learning. As a brand new research field, mobile learning can supply the learners with required knowledge and information anywhere, anytime, that can realize the practically independent learning. And as an important component of mobile learning, the research on mobile platform plays a vital role in this process.

Mobile Learning originated from 1994, the Wireless Andrew Project proposed in Carnegie Mellon University, which lasted three years. It finally constructed the wireless infrastructure to supply the high speed wireless connection for all the students, teachers in this University. Then, global

This work was financially supported by the Teaching Reform Research Project of Undergraduate Colleges and Universities of Shandong Province (2015M136); SDUST Young Teachers Teaching Talent Training Plan; SDUST Excellent Teaching Team Construction Plan; Teaching research project of Shandong University of Science and Technology (JG201509 and qx2013286); Shandong Province Science and Technology Major Project (No. 2015ZDXX0801A02). mobile learning began to carry out. In abroad, mobile learning mainly aims at developed countries and areas with broader width and greater depth. Mobile learning can be categorized into two kinds, first kind is proposed by the e-Learning providers, who try to borrow the e-Learning experience, and broaden the market of m-Learning[1], which is mainly exploited in enterprise training; the other kind is presented by educating organization based on school education, and tries to introduce new techniques in teaching, learning and managing. In Europe, the "From Digital Learning to Mobile Learning" project supported by the Da Vinci Project and organized by Ericsson educating organization, aims at implementing the mobile communication technology to promote and advance professional education and training reformation. The European M-Learning, proposed and hosted by British ISDA [2], this project aims at developing a prototype product, which can supply mobile learning information and content, stimulate the enthusiasm of learners, and increase the employment opportunity. Stanford University learning lab (SLL) [3] introduced the mobile terminal exploited in business field, tries to transits the mobile terminal to in-class language teaching. They invent the mobile learning module for foreign language learning, which can be used to learn foreign language. On the other hand, the British Birmingham handler mobile learning project, Finland Tampere University's X Task mobile learning system, the Norway Oslo University's KNOWMOBILE project are all sponsored by the government, academy, and University.

At present, the research on mobile learning platform mainly focuses on cell phone information based mobile learning system, WAP based mobile learning website, and the $\mathrm{C} / \mathrm{S}$ based mobile learning platform. For example, the WAP based mobile learning website of Shenzhen Broadcasting Television University[4,5], impressed the students with its availability and practicality, they all admitted that the system can satisfy their requirement anytime, facilitate the communication among the students and teachers timely and smoothly, and a beneficial complement for digitally learning with website. The IOS and Android based mobile learning platform developed by 
ShangHai Jiao tong University, which implements the video learning, online discussion, live broadcasting, etc. In abroad, the intelligent terminal based mobile learning platform Blackboard Mobile developed by Blackboard Corporation in America [6], which can carry out teaching and learning activity anywhere, anytime, it can support IOS, Android, and Web OS. Furthermore, it can also receive course activity related messages, browse course homework and score, and publish the discussion and mobile testing result.

Although the above mentioned mobile learning platform can solve some present problems in traditional in-class teaching procedure, but some other problems still exist, such as: 1) the learning content aspect, the diversity is not enough for different specialized content. 2) the mutual interaction of present mobile learning platform, the interaction among the students and teachers is monotonous, which mainly are message and email, etc. 3) the learning tools and corresponding support, as the technique is growing rapidly, how to support the novel mobile devices with our mobile learning platform needs to be considered. The Electrical Engineering and Automation is one of the traditionally advanced and specialized subject in Shandong University of Science and Technology, it is also one of the important subject group in our University. From 2010, approved by the Education Administrative department, our University and Tasmania University in Australia began to carry out the cooperative program of "Electrical Engineering and Automation". To our best knowledge, in Tasmania University the raising objective of this subject is established on the basis of "Big Engineering", which requires that the students should satisfy the requirement of both academic and engineering request of Australian engineer association; meanwhile the students should also have thorough professional knowledge and skills. As the course content is different from the Chinese current raising plan and course architecture, and the relative specialized courses are closely interconnected. Because of the confinement of semester setting and teacher resource, the in broad courses are relatively independent, loosely connected, which asks the students to comprehend not only the traditional in-class knowledge, but also the independent learning outside of the classroom to fulfill the requirement of practical work.

And in 21st July 2014, Chinese CNNIC published the 34th investigation report, which shows that until then on, there are more than 632 million persons have bene on the Internet, and 527 million of them are using mobile phones. In September, according to the data by Internet real-time statistics, there would be more than 3 billion persons in Internet. The quantity of the Internet users brought the astonishingly rapid development of Internet, from 1969 the invention of Internet till now, less than 45 years but still penetrate the whole world. Internet not only affects everyone in this world, in 1991 the Professor Kevin Ashton from MIT proposed the thing of Internet conception, even the things surrounding us has been connected into Internet. Intelligent mobile phone, smart watch, smart glass, they all can produce real-time data of persons who are wearing them. Internet once again permeates our lives. So many Internet users and things, the data produced everyday would be a much bigger number. In nowadays, the data produced in Internet is valuated by EB, which is way more than the data produced in the history of human beings, including all of the words spoken by all of the human beings in the history. So much data are not garbage like or meaningless collection, the information in these data are highly valuable, through which all of the content can be extracted with analyzing and mining. Big data has become one novel resource, following the rapid growth of Internet technology, the acquisition and storage of data is decreasing rapidly. Through Big Data, we can analyze the learning behavior, habit, and interest of students. Professor Victor, Mayer-Schnberger [7], who's is called after the prophet of the Big Data era, is also studying in Oxford University, Networking Academy, he considered that the era of Big Data has come, and new thought will replace the old thought finally, and the Whole Data will replace the Random sample, hybrid is more important than precision, and focus on correlativity rather than causality. Meanwhile, the memory characteristics of Big Data era should invoke the relative thought of human beings, if we should memorize all of the things permanently, or follow the nature of human beings to settle an expiration of memory. In the research of Victor Michael-Schnberger, the security of data should be paid more attention, in the flood of big data, data and information security should play a more important role. As a new producing material, the possession and controlling of data is one of his research interests. The accessibility, durability, and memorability would cause the inequality of data controlment. The gap between rich man and poor man of information will be wider. Thus our mobile learning platform should supply the analyzing function of Big Data.

In order to supply the students with a beneficial independent learning environment, we need to construct a mobile learning based independent learning platform. Based on the above mentioned reasons and combined with the practical situation of the cooperation between Shandong University of Science and Technology and Tasmania University, we present the fundamental functions and integrated architecture of a mobile learning based independent learning platform, and give suggestions to the other subjects in our University.

\section{FunCTIONAL ANALYSIS ON MOBILE LEARNING BASED INDEPENDENT LEARNING TERMINAL PLATFORM SYSTEM}

We collect the fundamental functional requirements of the users in plenty of ways, and reorganize the learning terminal platform users to be students' users and teachers' users, according to the characteristics of independent learning; the whole learning terminal platform should have two fundamental functions: independent learning function and mutual learning function, which are described as follows:

\section{A. Independent learning module}

Independent learning module is the fundamental module of any learning platform, thus we begin with analyzing it. The flow of learning online with the learning platform of Shandong University of Science and Technology includes three steps: firstly the student should input their own student ID to log into the system, followed by choosing subject, choosing course, choosing specific learning content. For example, for the students of Electrical Engineering and Automation, the default user should be from it, then they should choose the course unit, such as: Circuit, Simulated electronic technique, digital 
electronic technique, automatic control theory, Power station and power system, about 15 courses are taken as their main courses, and optional courses should increase in the following stages in the future. And finally they should pick up the content.

The representation of the online learning should be diverse, which can represent the learning content of each subject. At present, most of the MOOC kinds of online learning system adopt three screens to record their teaching material. And the three split screens mean they divide the PowerPoint into three zones: video zone, view tree zone, and documentation zone. The first zone is used to play the video of teaching scene or scenario; the view tree zone is exploited to show the chapters or menu, the learners can independently choose relative chapter during the learning process; the documentation zone is used to play the video and relative file, or to switch the picture, text according to the content. The advantage of this rendering method is the streaming technique, which is not only mutually but also ampler. But mobile learning based independent learning terminal platform system need to consider the habit of the learner. Firstly, the transaction period should be as short as possible, the mobile user can not execute a great deal data or tasks, and the period of these tasks should be short, too. The user always wishes to finish their task as soon as possible. For example, the online reading application should supply the users with a general structure, which can be by the users to access the content easily and change the reading position frequently. Secondly, we should consider the concentration of the user. Mobile learner usually does not sit by table to use their phone; otherwise they use the phones when they travel around or visit by bus. Thus the learning resource should be with more pictures, which can attract the user. Meanwhile, the performance of the mobile learning resource must be optimized, the mobile use prefer the response time shorter the better. If the response time is too long, it would not only affect the performance of specific component, it would also affect the exploitation of the whole system. Finally, we should pay more attention to the confinement of the persons' sensing system. In the communication system, each channel is responsible for receiving and sending specific kind of information. And channel is the path of communication between receiver and sender. The mobile learning platform has multiple user interfaces; we should avoid designing the overloaded information processing ability. Thus, this kind of PowerPoint is not suitable for vision limited mobile terminal. Because at present the WAP based mobile learning website is transferring the HTML described information to WML based information, and shows the information on the screen of mobile phone or other hand hold device, which may omit some important information and affect the integrity and mobility. According to the above analysis, and considering the requirement of mobile learning, this system adopts the design of reading device, which reads the website content through tree structured menu; it can support the reading of both HTML and XML. The learner can independently choose the learning content, which requires less resource and less bandwidth.

\section{B. Mutual learning function module}

In the first section, we present that in the current mobile learning platform the communication among the students and teachers are traditional BBS based pattern. And the communication based on BBS, requires the timely refreshing mechanism on Web page, which is not suitable for mobile learning. And for the cell phone users in China, the refreshing mechanism would definitely increase the cost of them. The mutual learning functional requirement is separated into realtime mutuality and non-real-time mutuality two sub-modules, which can satisfy the users with different levels. The mutual learning is one of the most important parts in mobile learning platform, the discussion and communication is implemented by this module. Real-time mutuality is used to realize the common discussion about general problem or question, which focuses on the time effectiveness of information transferring. On the other hand, non-real-time mutuality is used to implement the communication among the students and teachers, which aims at increase the suppleness of the content and the convenience. To be the best, these functions should combine with the communication methods in Internet including Email, Forum, WeChat voice, etc.

\section{The analysis of student learning behavior based on Big Data.}

The routine of the students' learning and the data of thought can be extracted by mobile learning platform. The data can satisfy $4 \mathrm{v}$ elements of Big Data. Thus, we supply the analyzing method based on Big Data on our platform to analyze the behavior of students, and delve into more detailed information including the learning status, knowledge, and supply the customized learning.

\section{SUGGESTIONS TO THE FUtURE CONSTRUCTION OF Mobile LEARNING PlatForm}

Through the analysis on mobile learning resource construction of the Electrical Engineering and Automation by Tasmania University and our University, we present some suggestions for the construction of mobile learning platform.

\section{A. Construct a learning management system to store the mobile learning content.}

In the future, most of the teachers in University would store their teaching materials in a specific learning management system, which can be used to support the students. Including the textbook, the PowerPoint and relative in-class videos, the students can find plenty of useful materials in this system. Thus at the beginning of the construction of this mobile learning platform, we should firstly consider of building a learning management system with diverse learning content.

\section{B. Build a learning analyzing system based on Big data.}

When the students enter the mobile learning platform, the system should present the statistics to them, such as the most viewed resource, most recent resource, and other relative data or flowchart. For example, if the status of specific student can be captured by the system, then the system would supply them with content that can satisfy their requirement dynamically. When some student stays in one learning status for a long time, the system can decide if the student has met some kind of difficulty, then it can give the student some hints about the learning material to assistant the student. Meanwhile, in personal learning environment, to analyze the learning activity 
record daily, weekly, monthly, or annually can present us more useful information. The information would increase exponentially, including the social information, blog message, email, learning status, etc. Above mentioned factors require the big data analysis system. The Education researcher and organization all can carry out science research with these data.

\section{Enhance the self adaptability of the mobile devices.}

The mobile devices would render different performance to the same mobile learning platform and different user. This needs us to enhance the adaptability of the mobile learning platform to break the temporal and spatial confinement, and supply with timely required learning.

Above all, the mobile learning plays an important role in recasting the higher education learning. No matter concentrating on pure online course, mixed course, or making use of the online learning management system to implement the face-to-face teaching and experiment, the students can learn anytime, anywhere. Although the networking technique has changed the learning method, we still should notice that right now it is a complementary content to the current structure of University; the fundamental structure of our University has not changed thoroughly.

\section{CONCLUSION}

Mobile Learning cannot amplify the methods of acquiring knowledge, constructing knowledge, applying knowledge, it also can change the traditional learning thought, and supply life-time learning, universe learning, and customized learning with strong support, and finally it can implement the independent learning. This paper aims to analyze the key functions of independent learning platform based on the specialty of the Electrical Engineering and Automation by Tasmania University and our University, and then we present some suggestions about the construction of independent learning platform. First, we should construct a learning management system to store the mobile learning content. Second, we should make use of the big data to analyze the learning. Third, we should enhance the self-adaptability of the learning tools.

\section{REFERENCES}

[1] M-learning[EB/OL], http://www.m-learning.org/index.shtml.

[2] J. Attewell, "Mobile technologies and learning: A technology update and m-learning project summary", Technology Enhanced Learning Research Centre, ISBN 1-84572-140-3.

[3] Stanford Learning Lab [EB/OL], http://sll.stanford.edu/projects/mobilele arning.

[4] Hu Xiaohong, "Track five examples of mobile learning in the world," Chinese Distance Education, No.2X, 2005, pp. 49-51. In Chinese.

[5] Yao Yumin, "Mobile Learning Based on Mobile TV Technology," Joournal of Hunan Radio and Televistion University, No.1, 2010, pp. 53-55. In Chinese.

[6] Blackboard Mobile [EB/OL], http://www.blackboard.com/platforms/mo bile/overview.aspx

[7] Viktor Mayer-Schnberger, "Big Data: A Revolution That Transforms Ho w we Work, Live, and Think", Houghton Mifflin Harcourt, 2012, ISBN 978-0544002692 